\title{
Entidades socialmente creativas en un contexto de gobernanza multinivel. Una comparativa del fomento de la economía solidaria en Barcelona y Bilbao
}

\author{
Santiago Eizaguirre Anglada \\ Universidad de Barcelona \\ santieizaguirre@ub.edu
}

Recibido: 19-12-2013

Aceptado: 29-07-2015

\section{Resumen}

En este artículo, se ahonda en el conocimiento sobre el enraizamiento territorial de los procesos de innovación social. La investigación se centra en la participación en la gobernanza de dos redes de entidades (la Red de Economía Alternativa y Solidaria - Euskadi, en Bilbao, y la Xarxa d'Economia Solidària de Catalunya, en Barcelona) que se orientan hacia el fomento de la economía solidaria, centrando su actividad en dos ciudades distintas: Barcelona y Bilbao. Se parte de la hipótesis de que es posible identificar a estas redes como entidades socialmente creativas — precursoras de dinámicas de innovación social—, que trabajan para producir cambios en las dinámicas de exclusión social y las relaciones entre grupos sociales. Ambas se caracterizan por ser organizaciones complejas que consideran las dimensiones económica, cultural y política de la transformación social. Se observa en detalle cómo estas redes articulan las relaciones de gobernanza con las administraciones públicas, así como los mecanismos a partir de los cuales consideran que son capaces de promover innovación política. En la consideración de la utilidad de la participación en la gobernanza con las administraciones públicas, se presentan diferencias sensibles entre ambas. La investigación concluye que los sistemas locales de gobernanza multinivel — con sus culturas políticas particulares de negociación y conflicto- condicionan la organización de las dinámicas de innovación social. Se expone el modo en que la materialización respectiva de las relaciones de gobernanza en Barcelona y en Bilbao imprime un carácter específico al asociacionismo socialmente transformador y define unas condiciones particulares para la innovación social. Palabras clave: innovación social; gobernanza multinivel; economía solidaria; sociología urbana 
Abstract. Social innovation and local systems of governance: A comparison of political participation by networks for the promotion of a solidarity-based economy in Barcelona and Bilbao

This article deals with the knowledge on the territorial rooting of social innovation processes. The research focuses on the participation in governance of two networks of entities (Red de Economía Alternativa y Solidaria Euskadi and Xarxa d'Economia Solidaria de Catalunya) aimed at promoting a solidarity-based economy, which focus their activity on two different cities: Barcelona and Bilbao. It starts from the assumption that it is possible to identify these networks as socially creative initiatives which promote dynamics of social innovation and are working to produce changes in the dynamics of social exclusion and the power relations between social groups. Both entities are characterized as complex organizations that consider the economic, cultural and political dimensions of social transformation. It is shown in detail how they articulate governance relations with public authorities and which mechanisms they consider capable of promoting innovation in policymaking. Significant differences between the two selected cases are found regarding the utility of participating in governance relations with public administrations. The research concludes that local systems of multilevel governance - with their particular political cultures of negotiation and conflict - condition the organization of social innovation dynamics. The article reveals how the materialization of governance relations in Barcelona and Bilbao lend a specific character to socially transformative associations and define particular conditions for social innovation.

Keywords: social innovation; multilevel governance; solidarity-based economy; urban sociology

\begin{abstract}
Sumario
Introducción

El fomento de la economía solidaria en Barcelona y Bilbao

El énfasis en la innovación social en un Conclusiones

Barcelona y Bilbao, narrativas de Referencias bibliográficas transformación socioeconómica y prácticas de ciudadanía

\section{Introducción}

En este artículo, se presenta una exploración sobre el modo en que las ciudades, en tanto que sistemas locales en los que se materializan relaciones de gobernanza multinivel, condicionan las dinámicas de innovación social promovidas por la ciudadanía organizada. Con el uso de las expresiones dinámicas de innovación social y estrategias o entidades socialmente creativas, nos referimos, a lo largo de estas líneas, a la acción y al efecto de prácticas promovidas por entidades de la sociedad civil específicamente organizadas para el tratamiento y la transformación de las relaciones de poder que inciden en la definición de pautas de desigualdad y exclusión social. Más allá del asistencialismo o del ofrecimiento de recursos y servicios a personas que no pueden ver satisfechas sus necesidades mediante los mecanismos que ofrece el estado o el mercado, la
\end{abstract}


innovación social (y, por ende, a lo largo de esta investigación, hemos identificado a lo que consideramos estrategias socialmente innovadoras o creativas) requiere también de una atención al empoderamiento cultural de los lenguajes para hacer referencia a las situaciones de exclusión social, así como a la acción política orientada a la transformación de las relaciones de poder que inciden en la generación de dinámicas de desigualdad.

Desarrollando un análisis de estrategias socialmente creativas en las mismas áreas de innovación social en dos ciudades distintas, Barcelona y Bilbao, hemos querido destacar el papel clave de los sistemas locales de gobernanza en la definición de dinámicas de innovación social. Para caracterizar cada una de estas ciudades en tanto que sistemas locales, es relevante desarrollar indicadores comparativos sobre la economía urbana, la política social y la cultura política de cada una de ellas como variables territoriales clave que afectan al desarrollo de las dinámicas de innovación social. En este artículo, nos centramos en la comparativa de la última de estas tres grandes variables: la cultura política de negociación y conflicto entre las administraciones públicas y el asociacionismo socialmente transformador. Las estrategias institucionales de transformación socioeconómica en un contexto postindustrial como uno de los posibles indicadores de la economía urbana o las arquitecturas institucionales particulares para hacer frente a la exclusión social como indicador de las políticas sociales también son cuestiones clave para contextualizar las dinámicas de innovación colectiva. En este artículo, haciendo referencia a la cultura política, apuntamos a las singularidades locales de la historia de movilización, negociación y conflicto entre administraciones públicas y entidades ciudadanas como uno de los indicadores clave en la comprensión sobre el modo en que las ciudades y los territorios, en tanto que sistemas locales, pueden condicionar la innovación social.

Los datos que se presentan han sido recogidos entre los años 2009 y 2012, principalmente mediante el análisis cualitativo de 54 entrevistas en profundidad con informadores privilegiados: representantes de organizaciones de la sociedad civil, técnicos de las administraciones públicas y académicos involucrados en el estudio de las políticas sociales en Barcelona y Bilbao. También se ha realizado un estudio cuantitativo de datos financieros sobre un conjunto de 20 entidades identificadas como «socialmente creativas», 10 en cada ciudad. Este análisis se ha centrado en la observación de datos, tanto en los referentes a sus fuentes de ingresos como a la estructura de su gasto ordinario.

El artículo consta de tres partes diferenciadas y unas conclusiones. Primero, se arranca la discusión situando el debate durante la primera década del siglo XXI alrededor de la noción de innovación social desde la perspectiva específica de los estudios urbanos y los análisis de gobernanza. Se argumenta cómo el cambio en las formas de gobernar y los retos generados por la necesidad de desarrollar el trabajo en red entre administraciones públicas y sociedad civil han promovido la presencia, en el discurso político, de la noción de innovación social. Desde la perspectiva teórica de los estudios de gobernanza urbana, este empeño se puede relacionar con la necesidad de realizar un desarrollo 
sustantivo de los mecanismos de toma de decisiones participada por distintos actores, así como con la necesidad de comprender los entresijos de la participación ciudadana. La reflexión académica y la narrativa política alrededor de la innovación social y lo socialmente creativo se ha producido de manera paralela al desarrollo de una comprensión institucional cada vez mayor entorno de la necesidad de estimular la implicación de la sociedad civil, además de los usuarios de las políticas públicas, en la búsqueda de respuestas y de soluciones a los retos generados por unas sociedades cada vez más desiguales y complejas. Las implicaciones en términos de externalización de las responsabilidades que puede tener un énfasis determinado en las dinámicas de innovación social, sin considerar la importancia de un papel redistributivo y regulador por parte de las administraciones, es el punto crítico que se señala en este debate.

En segundo lugar, se procede a la presentación del modo en que los sistemas locales de gobernanza multinivel en los que se ha ubicado la investigación imprimen diferencias en las características del tejido asociativo socialmente transformador. Barcelona y Bilbao son ciudades que han vinculado sus narrativas de transformación socioeconómica postindustrial al discurso de la innovación social y, pese a sus semejanzas en este aspecto, difieren en el modo que fomentan las dinámicas promovidas contra la exclusión social por parte de la ciudadanía organizada. En este apartado, se hace hincapié en el desarrollo de sus procesos respectivos de transformación socioeconómica ocurridos principalmente durante la última década del siglo xx y en el modo en que han incidido en la construcción de una cultura política de negociación con la ciudadanía organizada por la transformación social. Se presenta la relación de estas dinámicas de transformación socioeconómica de tipo postindustrial con el desarrollo de estilos particulares por lo que se refiere a las relaciones de gobernanza de la Administración y la ciudadanía organizada.

El tercer apartado del artículo analiza cómo los caracteres diferenciados de los contextos particulares se relacionan con rasgos específicos en las organizaciones orientadas al fomento de la economía solidaria en ambas ciudades. Se presentan los rasgos generales de la actividad de la Red de Economía Alternativa y Solidaria - Euskadi, en Bilbao, y de la Xarxa d'Economia Solidària de Catalunya, en Barcelona. En esta comparación, se observan diferencias atribuibles a los estilos particulares de gobernanza multinivel con los que cuentan ambos contextos y que se materializan, entre otras cosas, en las formas de financiación pública con las que se respaldan iniciativas socialmente transformadoras promovidas por la ciudadanía organizada.

Las conclusiones del artículo recogen las observaciones señaladas en los apartados anteriores, destacando la naturaleza socialmente construida y territorialmente enraizada de las dinámicas de innovación social.

\section{El énfasis en la innovación social en un marco de gobernanza multinivel}

El estudio comparativo de la innovación social promovida por la ciudadanía en un marco de gobernanza comparada es uno de los objetos de análisis en 
los que los estudios urbanos dialogan con la sociología política y la geografía económica. Su interés se puede relacionar con la identificación previa de un proceso de reestructuración de las funciones del estado, que atribuye un reconocimiento creciente a la importancia de la esfera regional y urbana en la toma de decisiones, especialmente las relacionadas con las políticas sociales (Brenner, 2004; Garcia, 2006; González y Healey, 2005).

Hay un cuerpo literario en ciencias sociales muy activo durante la primera década del siglo xxI que pone el enfoque en el cambio en las formas de gobernar desde un modo de proceder centralizado y autoritario hacia un rol mediador y facilitador (Brenner, 2004; Jessop, 2002, 2004; Le Galès, 2002; MacLeod, 2001; Rhodes, 1997). En un contexto de competencia urbana que trasciende las fronteras de los estados nación, el interés de las administraciones públicas en desarrollar alianzas con la sociedad civil ha sido analizado en profundidad por este cuerpo de literatura académica. En paralelo al incremento del interés en las asociaciones públicas y privadas, y ante la identificación de la estructura de redes como elemento crucial del desarrollo de la nueva economía, la gobernanza se convirtió, a lo largo de la primera década del siglo XXI, en una noción bien conocida para hacer referencia al desarrollo de colaboración entre administraciones y representantes de la sociedad civil, tanto desde el sector orientado al beneficio privado como el que no tenía ánimo de lucro (Geddes, 2006).

Este proceso de atención al rol descentralizado y coordinador por parte de las administraciones públicas ha ido en paralelo a la identificación del condicionamiento multiescalar de las políticas públicas, especialmente en los campos de la política social. La incidencia de las escalas supranacionales como la Unión Europea, además del análisis de gobernanza de las escalas subnacionales, ha devenido una variable clave para explicar las disparidades territoriales en las políticas de bienestar, especialmente en países federales o semifederales.

En orden a hacer frente a la competencia urbana y el declive postindustrial, los gobiernos locales en ciudades como Barcelona y Bilbao se vieron forzados a desarrollar estrategias públicas para promover la consideración de sus ciudades y regiones como centros atractivos en la nueva economía. Esto ha implicado el desarrollo de políticas que han buscado la reconversión de antiguos centros industriales en economías de servicios basados en el conocimiento. A pesar de que Bilbao inicia este proceso de transformación con posterioridad a Barcelona, a partir de los años ochenta en Barcelona y desde los años noventa en Bilbao, en ambas ciudades, hay una búsqueda de la transformación de una economía industrial hacia una economía basada en el conocimiento.

Además, en paralelo con la preocupación institucional sobre la transformación económica, es posible identificar un cambio en la terminología utilizada por las administraciones públicas para hacer referencia a los retos sociales. Un nuevo énfasis en la gobernanza es promovido institucionalmente buscando la colaboración de actores de la sociedad civil en la aplicación de respuestas a los retos más actuales. Esto se articula en relación con un cambio en los modos de atender a la desigualdad social. La pobreza no se concibe ya como 
una condición estática y empieza a ser reconocida, a finales del siglo xx, como una condición dinámica y relacional. La noción de exclusión social ofrece una explicación multidimensional, y en ocasiones fragmentada, de la desigualdad social. Durante la primera década del siglo Xxi, palabras resbaladizas del tipo cohesión social o capital social se difunden en el campo de las políticas municipales, subrayando la transformación de la atención institucional a aspectos como la participación ciudadana o la diversidad, sin resolver al fin las causas que producen la pobreza ni las dinámicas de exclusión social (Maloutas y Pantelidou, 2004; Sommers, 2005). Siguiendo este cambio en las maneras de hacer referencia a la diversidad y a la desigualdad, podemos afirmar que la gobernanza participativa y la innovación social son, en la actualidad, las dos principales áreas de interés en la escena de la investigación urbana que recogen los debates citados.

La investigación sobre gobernanza participativa y deliberación pública trata la cuestión relativa sobre el modo de llevar a cabo la democracia directa en un contexto de diversidad y de complejidad creciente. Las potencialidades de la gobernanza participativa, además de sus contradicciones y paradojas, han obtenido atención entre los temas más debatidos en los estudios urbanos durante la primera década del siglo Xxi (Blakeley, 2010; Casellas, 2007; Silver et al., 2010, Parés, 2009). Este énfasis ha ido en relación con la progresiva institucionalización de procesos participativos como un mecanismo usual en la gobernanza local.

Una parte significante de la investigación sobre participación aplicada a la escala local prueba de entender cómo las personas, los representantes de la sociedad civil y los responsables de la Administración pública colaboran y consiguen llegar a cierto grado de acuerdo. Este cuerpo de literatura, identificado por algunos de los revisores de la literatura como la perspectiva "habermasiana», hace énfasis en la búsqueda de una situación ideal en la comunicación entre representantes de la ciudadanía y las administraciones públicas. Desde esta perspectiva, la democracia participativa y deliberativa son estudiadas desde un punto de vista pragmático, tratando de entender las condiciones para un procedimiento óptimo orientado a resaltar la democracia local (Beaumont y Nichols, 2008; Bifulco y Centemeri, 2008; Blanco y Gomà, 2003; Fung, 2004; Gbikpi y Grotte, 2002; Silver et al., 2010; Uitermark y Duyvendak, 2008).

De modo complementario, existe un cuerpo de literatura que argumenta la imposibilidad de la existencia de un choque de intereses igualitario y la existencia de márgenes silenciados en todos los procesos participativos (Mouffe, 2000; Swyngedouw, 2005). Esta literatura subraya la narrativa neoliberal inherente que va de la mano con el énfasis actual en la participación desde una perspectiva basada en la construcción de consensos. Desde este punto de vista, identificado por algunos autores como la perspectiva "foucaultiana» (Silver et al., 2010), el énfasis del análisis tiene que situarse en las relaciones de poder, concibiendo críticamente los procesos de desactivación y cooptación de las perspectivas críticas que se derivan de una aproximación de arriba hacia abajo a la participación ciudadana. Esta es una aproximación que promueve 
una mirada a los márgenes silenciados que emergen en todos los procesos de participación (Beaumont, 2008).

De modo paralelo al reconocimiento de la participación ciudadana como clave en la gobernanza multinivel, la innovación social ha sido promovida en el ámbito internacional como un objetivo crucial para las sociedades urbanas (Drewe et al., 2008; MacCallum et al., 2009; Moulaert et al., 2013). Las narrativas oficiales han tendido a desarrollar el concepto como un valor clave en orden a facilitar la lucha contra la fragmentación social y la revitalización económica. Esto se ha producido en un contexto de decrecimiento del gasto público que incide en subrayar la sospecha de que el uso de la expresión se entiende como un modo de externalización de la responsabilidad sobre la política social a la sociedad civil.

Obviando el papel clave de la Administración pública, los actores de la sociedad civil son enarbolados como los agentes más apropiados para tratar la fragmentación social. En el marco de la promoción discursiva de la idea de innovación social, la participación de organizaciones de la sociedad civil orientadas al tratamiento de la desigualdad en la gobernanza de la exclusión social tiende a ser considerada un objetivo a conseguir por parte de las administraciones públicas. Este rol atribuido a las organizaciones de la sociedad civil que se ocupan del tratamiento de las dinámicas de exclusión social es reforzado, por ejemplo, cuando las administraciones públicas enfatizan el rol de los actores del tercer sector en la aplicación de las políticas sociales. Pero, ¿en qué sentido podemos afirmar que las organizaciones de la sociedad civil que afrontan cuestiones vinculadas con la desigualdad son suficientemente autónomas e independientes para tratar con los retos sociales por su cuenta?

Lo que pretendemos subrayar con esta pregunta es que, en muchos casos, la innovación social es utilizada como un eufemismo para hacer referencia a una forma barata de hacer frente a la fragmentación social. Benoît Lévesque (2013) y otros autores han observado cómo el interés creciente por la innovación social desde la perspectiva de las administraciones públicas se puede explicar bajo la perspectiva de una nueva ola de reformas inspiradas por el pensamiento neoliberal vinculado a la nueva gestión pública. En este sentido, es frecuente la promoción de la innovación social como una alternativa a la inversión pública y el gasto social en políticas redistributivas. La innovación social es referida como una política desarrollada por las organizaciones de la sociedad civil y por parte de empresas privadas o de los propios usuarios de los servicios y de los recursos públicos. Desde esta perspectiva política, la promoción de la innovación social implica la transferencia de responsabilidad de tratar las dinámicas de exclusión desde el gobierno hacia lo que Erik Swyngedouw define como la gobernanza por debajo del estado (Swyngedouw, 2005).

Ante esta utilización interesada del concepto, lo que Erik Swyngedouw define como una cuestión clave es la definición de lo socialmente creativo. ¿Qué entidades de la sociedad civil organizada son socialmente innovadoras y cuáles no? A esta pregunta, la literatura desarrollada alrededor de los estudios urbanos ha promovido una respuesta inequívoca. Las dinámicas de innovación 
social, o lo mismo se entiende, las entidades o iniciativas socialmente innovadoras, se pueden caracterizar por su énfasis en la producción de cambios en las relaciones de desigualdad entre grupos sociales (Moulaert et al., 2013; Defourny y Nyssens, 2013). En concreto, la definición que más fortuna ha hecho dentro de los estudios urbanos del concepto de innovación social se basa en tres dimensiones básicas de atención a las dinámicas de exclusión social (Moulaert et al., 2013). En primer lugar, se refiere a la acción y al efecto de mecanismos redistributivos que no son garantizados ni por el estado ni por el mercado. En segundo lugar, hace referencia al desarrollo de empoderamiento cultural o a los lenguajes críticos para que los sujetos o los grupos sometidos a dinámicas o a riesgo de exclusión puedan subvertir su situación. Finalmente, en tercer lugar, y esta es la dimensión más compleja del fenómeno, la innovación social implica la producción de cambios políticos en la esfera pública referentes a las relaciones de poder que inciden en la desigualdad entre grupos sociales. Estas son las características que deberíamos encontrar en las dinámicas de innovación social y lo que podemos llamar entidades, iniciativas o estrategias socialmente innovadoras. El problema teórico que surge a continuación es que son muy pocas las experiencias que puedan ser ejemplares a la vez en las tres dimensiones presentadas, ya provengan de la acción de las administraciones públicas o de la ciudadanía organizada.

\section{Barcelona y Bilbao, narrativas de transformación socioeconómica y prácticas de ciudadanía}

Observando la historia de los procesos de transformación urbana más recientes en Barcelona y Bilbao, es posible identificar un primer estadio en que el planeamiento urbano es concebido como un instrumento orientado a mejorar las condiciones de vida de la población, dotando a esta de un sentido «ciudadano". El llamado urbanismo ciudadano, orientado a proveer de equipamientos y servicios a todos los barrios de Barcelona, es especialmente activo durante los años ochenta y se aprovecha del anuncio, en 1986, de la organización de las olimpiadas por parte de la ciudad como una ocasión para desarrollar grandes inversiones en ella (Borja, 2004; Capel, 2005; Degen y Garcia, 2008; Montaner, 2004). En una línea similar, durante los años noventa, en Bilbao, varias grandes operaciones de inversión pública se orientan a la promoción de infraestructuras urbanas y a la remodelación socioeconómica postindustrial. La remodelación de la Ría y la construcción del metro, así como la renovación de la estación de tren o del aeropuerto, han sido considerados logros de un urbanismo que se traduce en una mejora de las condiciones de existencia, la autoestima y el dinamismo de la ciudadanía bilbaína (Cenicacelaya, 2004; Urrutia, 2004). Podríamos hablar, pues, de ejemplos de un urbanismo «socialmente creativo" que empodera a la ciudadanía para abordar el cambio en las condiciones de vida que conducen a la exclusión y reproducen la desigualdad.

Pero, pese a las narrativas de transformación exitosa que se fomentan desde las instituciones durante esos años, hay relatos alternativos que ponen en duda 
la traducción de los procesos de transformación urbana de ambas ciudades en términos de equidad y de justicia social. En Barcelona y en Bilbao, emergen voces críticas por lo que se refiere a las estrategias urbanas de remodelación como una fuente de redistribución socioeconómica. Durante la primera década del siglo XXI, haciendo referencia explícita a ambas ciudades, se publican diversas obras que critican una deriva excluyente de las narrativas de transformación urbana barcelonesa (Andreu, 2008; Unió Temporal d'Escribes, 2004; Borja y Muixí, 2004; Capel, 2005; Delgado, 2007) y bilbaína (Cenicacelaya, 2004; Esteban, 2000; González, 2006; Plaza, 2006, 2008; Rodríguez, 2002).

A pesar de que esas críticas son relativamente simétricas en ambas ciudades, hay algunas diferencias entre los resultados obtenidos en el proceso de reconversión de una economía orientada a la industria a una economía orientada a los servicios. La coordinación de una alianza multinivel de administraciones públicas ha sido crucial en las dos urbes para iniciar el proceso de remodelación urbana, pero los mecanismos que han seguido ambos sistemas locales para conseguir la reconversión difieren de un contexto al otro. Mientras que, en el ámbito vasco, la mayoría de operaciones se han realizado sobre suelo público y han sido lideradas por las administraciones, en el ámbito catalán, el liderazgo de las agencias privadas ha sido mayor.

De modo paralelo a los procesos de transformación urbana y a la reconversión socioeconómica consecuente, el dinamismo de la sociedad civil, con sus especificidades concretas en lo que respecta a la cultura política de implicación en la gobernanza, también es importante para explicar la participación de las estrategias socialmente creativas en el tratamiento de las dinámicas de exclusión social. El tejido asociativo de Barcelona y Bilbao se caracteriza, durante los años noventa y a lo largo de la primera década del siglo XXI, por una diversificación de temáticas en torno a las cuales gravita la organización ciudadana. Durante la década de 1990, en ambas ciudades, hay un incremento considerable del número de asociaciones dedicadas especialmente a actividades culturales y de tiempo libre, una dinámica que ya había sido anticipada por autores que estudiaron el fenómeno del asociacionismo ciudadano haciendo hincapié en la década de 1980 (Domingo y Bonet, 1998; Urrutia, 1992). En la misma dirección, el estudio del incremento numérico de asociaciones y de otro tipo de entidades con finalidades no lucrativas subraya la idea de que el crecimiento del tercer sector social durante los primeros años del siglo XXI va en relación con el desarrollo de políticas de bienestar (ver figura 1).

Una cuestión importante para tomar el pulso de la colaboración entre asociaciones ciudadanas y administraciones públicas a nivel local es la relación entre movimientos vecinales y gobierno municipal. En ambas ciudades, los movimientos vecinales han tenido un papel crucial en la articulación de demandas con el fin de mejorar los recursos y la prestación de servicios. En la línea que definiría a la innovación social como un proceso desde abajo hacia arriba, en ambas ciudades, es posible encontrar ejemplos de los procesos promovidos por activistas vecinales que han conseguido compromisos institucionales por parte de las administraciones. 
Figura 1. Porcentaje de entidades no lucrativas respecto al total de entidades económicas. Barcelona y Vizcaya (1999-2009)

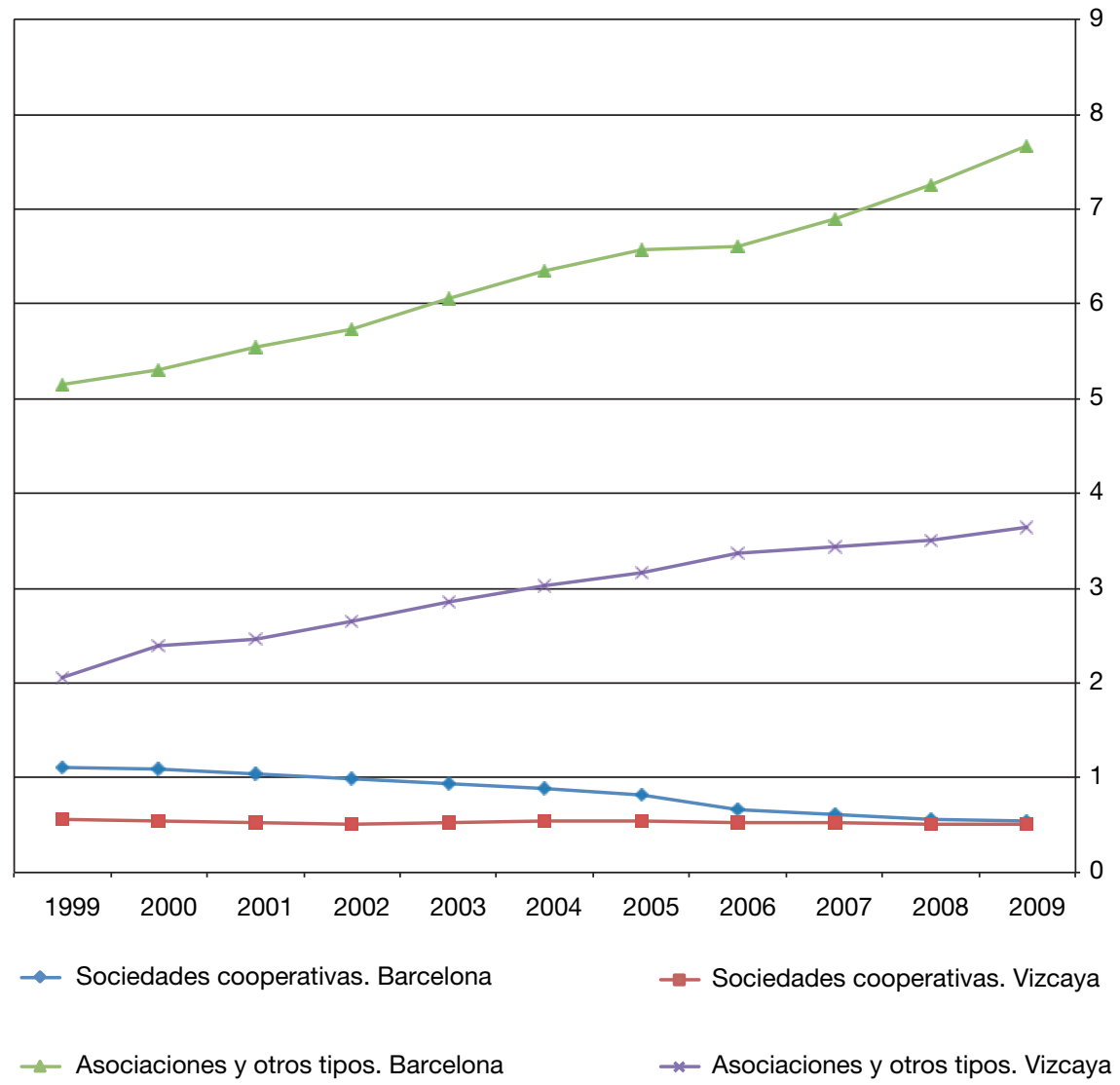

Fuente: elaboración propia. Directorio General de Empresas. INE, 2010.

Las relaciones entre los movimientos vecinales y las organizaciones públicas han evolucionado en las dos ciudades, desde una posición de clara confrontación (durante el período que va desde la legalización de las asociaciones cívicas, en 1964, hasta la recuperación de las instituciones democráticas, en 1979) hasta un cierto acuerdo sobre la institucionalización de canales de participación y una colaboración estable entre los ayuntamientos y los movimientos vecinales de ambas ciudades.

En la actualidad, hay, en ambas ciudades, varios ejemplos de equipamientos públicos con una gestión ciudadana que implica la existencia de un acuerdo entre el ayuntamiento y el movimiento vecinal. Este tipo de acuerdo ofrece apoyo financiero y atribuciones de toma de decisiones a las plataformas de residentes que reivindican el reconocimiento de su papel crucial (por ejem- 
plo: Ateneu Popular 9 Barris, en Barcelona, y Centro Cívico Otxarkoaga, en Bilbao). Esto puede considerarse un ejemplo del desarrollo de una relación de confianza entre el movimiento vecinal y las administraciones públicas. En este mismo sentido, en ambas ciudades, es posible identificar procesos de planeamiento estratégico que ofrecen un claro apoyo institucional a reclamaciones históricas de los residentes (por ejemplo: Pla Comunitari de la Trinitat Nova, en Barcelona, y Plan Comunitario Imagina Otxarkoaga, en Bilbao).

Pese a los ejemplos de acuerdo, el conflicto entre el Ayuntamiento y las redes socialmente transformadoras promovidas por asociaciones ciudadanas es una parte intrínseca de las relaciones de gobernanza urbana. En ambas ciudades, el conflicto se ha mantenido vinculado principalmente a la protesta contra operaciones concretas de planificación urbana o con el objetivo de exigir políticas sociales concretas. En Barcelona, durante la primera década del siglo XXI, se han producido varios episodios de ausencia de comprensión y confrontación entre las administraciones públicas y las redes ciudadanas. Estos episodios se han enmarcado en un ámbito tanto de ciudad (por ejemplo: las tensiones entre la FAVB, la organización del Foro de las Culturas en 2004) como de barrio (por ejemplo: Can Ricart en Poblenou o, más recientemente, el intento de demolición por parte del Ayuntamiento del Centro Social Okupado Can Vies, en el barrio de Sants). En Bilbao, el desalojo del Centro Social Okupado Kukutza, en septiembre de 2011, pone de relieve que, a pesar de que el régimen de bienestar vasco pueda ser considerado más desarrollado, su existencia no es garantía de atención a las dinámicas de innovación social promovidas por la ciudadanía, en particular las que parten de supuestos ideológicos explícitamente contrahegemónicos.

La fragmentación del tejido asociativo socialmente creativo es una dinámica común también en los contextos urbanos contemporáneos. La fragmentación que Enzo Mingione señaló a finales del siglo xx como propia de las dinámicas de exclusión en contextos urbanos (Mingione, 1991) puede extenderse también en las relaciones de gobernanza del tejido asociativo que se orienta a combatir la desigualdad social en las ciudades. En un marco conflictivo de cambio de época, es posible hablar de una transición de los actores que lideran la movilización de recursos, del paradigma de un movimiento social urbano tipo vecinal a un esquema más especializado en el que la implicación de las administraciones en la defensa y la legitimación de las estrategias socialmente creativas promovidas por la ciudadanía desempeña un papel clave. Las organizaciones que se orientan al tratamiento de un aspecto específico de las dinámicas de exclusión social son identificadas generalmente como tercer sector y son proveedoras de servicios públicos de las administraciones. Sin embargo, en muchos casos, también conservan características propias de los movimientos sociales urbanos, como organizaciones que se derivan de procesos de movilización de la ciudadanía, con una carga ideológica concreta y una dependencia organizativa de la militancia considerable.

Por último, también en este capítulo de similitudes entre Barcelona y Bilbao, cabe señalar que, en ambas ciudades, hay un proceso similar de institucionalización del conflicto social y la consolidación de las instancias representativas orientadas a facilitar la participación de la ciudadanía. En las dos existen 
instancias formales de participación orientadas a facilitar la descentralización de la toma de decisiones en el territorio por sectores temáticos de actuación. Además, tanto en Barcelona como en Bilbao, es posible encontrar una estrategia pública orientada a promover el asociacionismo. Por el contrario, cabe recordar que la existencia de acuerdos de participación ciudadana, así como políticas de fomento de espacios consultivos, no es una garantía de que las demandas de los ciudadanos, especialmente las relacionadas con el modelo urbano, se satisfagan directamente.

\section{El fomento de la economía solidaria en Barcelona y Bilbao}

Entre las entidades que se dedican a la promoción y al fomento de la economía solidaria ${ }^{1}$, se pueden distinguir dos grandes grupos: las que se dedican a la articulación de capital financiero para la consecución de proyectos de transformación social y las que se orientan a hacer presión política para el reconocimiento del sector, promoviendo el consumo responsable, el comercio justo y las redes de intercambio o los mercados sociales con valores solidarios y críticos. Aun compartiendo el objetivo común de impulsar la economía solidaria, ambos tipos de entidades tienen formas de funcionamiento claramente diferenciadas. Enmarcadas dentro del segundo grupo de entidades - las específicamente orientadas a la divulgación de la economía solidaria-, encontramos las redes de articulación institucional de todo el sector de la economía alternativa. Son redes que se ocupan de promover los valores del comercio justo, ecológico y sostenible, así como el consumo responsable y las prácticas empresariales que definen a la economía solidaria. En Bilbao, encontramos la Red de Economía Alternativa y Solidaria (REAS) y en Barcelona, la Xarxa d'Economia Solidària (XES), que responden a estos objetivos.

El modo en que estas plataformas o redes de entidades operan sobre las dinámicas de desigualdad social —aquello que hace que las podamos considerar socialmente creativas o innovadoras - se manifiesta de forma indirecta. Las redes de fomento de la economía solidaria, a diferencia de las empresas de inserción, por ejemplo, no ofrecen recursos desmercantilizados o servicios complementarios a los que ofrece el Estado que sirvan para la provisión de personas en situación o riesgo de exclusión social. Son las entidades que forman parte de ellas en tanto que integrantes de dichas plataformas las que responden a la dimensión productiva o redistributiva de lo que entendemos por innovación social. La aportación específica de las redes de promoción de la economía solidaria en términos de dinámicas socialmente innovadoras se refiere, principalmente, a la dimensión cultural y política del cambio social que llevan asociado.

1. Para caracterizar a los ámbitos de acción de la economía solidaria, Jordi Garcia, representante de la XES, habla de cinco ramas: la producción (que comprendería desde las cooperativas de trabajo asociado hasta las sociedades laborales $\mathrm{u}$ otras asociaciones que producen actividad económica), el comercio justo, el consumo responsable, las finanzas éticas y las redes de intercambio. 
En tanto que redes que estructuran el cambio cultural y político asociado a sus entidades integrantes, podemos considerarlas socialmente innovadoras. Inciden en el empoderamiento del sector promocionando el conocimiento respecto a los valores relacionados con el decrecimiento y las posibilidades que ofrece la economía pensada según el criterio del beneficio común. Son redes que posibilitan el desarrollo de lenguajes críticos y empoderadores. Por otro lado, en la dimensión política, institucional y organizativa, representan al sector delante de las administraciones advocando por la introducción de cambios legislativos que tengan en cuenta sus intereses, aunque, en este último aspecto precisamente, se presentan algunas diferencias entre contextos.

Una de las primeras cuestiones que emerge en la comparación entre la REAS Euskadi y la XES en Catalunya es que muestra una diferencia en la composición de la masa crítica que impulsa los planteamientos de la economía solidaria en ambos contextos. Si, en Euskadi, el fomento de la economía solidaria va muy ligado a las empresas de inserción, el cristianismo de base y la lucha contra la pobreza en contextos urbanos, en Cataluña, las entidades que fomentan de modo más comprometido los valores de la economía solidaria están especialmente vinculadas en un sector concreto del movimiento cooperativo especialmente comprometido con los ideales de transformación social.

Las diferencias en los tipos de actores que impulsan estas entidades pueden explicarse, entre otras cuestiones, por la trayectoria histórica del cooperativismo en ambos contextos. Mientras que, en el contexto vasco, el cooperativismo de trabajo asociado ha estado vinculado estrechamente con la perspectiva integral del grupo Mondragón ${ }^{2}$, muy activo y cohesionado y con renombre internacional, en el caso catalán, el cooperativismo y las sociedades laborales de trabajo asociado se rigen por una distribución geográfica dispersa que ha obligado al sector a realizar un esfuerzo para articular sus demandas mediante organizaciones sectoriales, como, por ejemplo, las federaciones de cooperativas. Ambas formas de articulación del cooperativismo, una más institucionalizada y otra más fragmentada, han tenido un efecto diferenciado en la constitución de las redes de fomento de la economía solidaria.

En el caso vasco, la REAS Euskadi nace con un carácter claramente independiente respecto al cooperativismo de Mondragón. La REAS Euskadi es una propuesta mucho más ligada a las entidades del tercer sector social que trabajan en la lucha contra la exclusión en el medio urbano, avalada y financiada por la Administración pública. La XES, en cambio, nace fruto del desarrollo de la sección de intercooperación de la Federació de Cooperatives de Treball de

2. Mondragón Corporación Cooperativa (MCC) es un grupo de cooperativas y empresas que constituye el primer grupo empresarial del País Vasco y el séptimo del Estado español, así como el mayor equipo cooperativo del mundo. Fue fundado por el sacerdote José Maria Arizmendiarrieta en el año 1956 en el pueblo de Arrasate (Guipúzcoa). En 2010, el grupo Mondragón estaba compuesto por 264 empresas y entidades que se dividían principalmente en tres sectores (financiero, industrial y de distribución), además de abastecer a las áreas de investigación y formación. En la actualidad, está teniendo lugar un intenso debate que cuestiona la estrategia de crecimiento e internacionalitzación de los últimos años. 
Catalunya y su vinculación con las administraciones, por tanto, es mediada por la federación y cuenta con una financiación pública sensiblemente menor que su homóloga vasca.

En el análisis de las relaciones de gobernanza, tanto de la REAS como de la XES, destaca que, a pesar de que, en ambos casos, se desarrollan prácticas innovadoras similares (por ejemplo: la popularización de las auditorías o el balance social), la posición respecto a las administraciones es diferente de una entidad a otra. Mientras la REAS Euskadi y también su entidad hermana GIZATEA tienen una postura proactiva muy clara respecto a las administraciones públicas, y especialmente respecto a la interlocución con los partidos políticos para la adopción de medidas, la XES destaca por la voluntad de no querer restar protagonismo a las federaciones de cooperativas y de no realizar tareas de representación política del sector. Esta diferencia, aparte de mediante las diferentes trayectorias históricas del cooperativismo en ambos contextos, también se puede explicar por la existencia de dos tipos de atención institucional a las redes de fomento de la economía solidaria muy claros ${ }^{3}$. Mientras el contexto vasco se caracteriza por una implicación prácticamente total de las administraciones públicas en el fomento de la economía solidaria y alternativa, dando una atención a la REAS Euskadi de entidad de interés público (ofreciendo el enlace permanente en la web del Departamento de Trabajo y Asuntos Sociales y la entidad, por ejemplo, o incluyendo el concepto de economía solidaria en la definición de objetivos del organismo del Gobierno Vasco encargado del fomento de la economía social), en el caso catalán, la atención de la Generalitat de Catalunya al trabajo realizado por la XES es prácticamente simbólica, por no decir inexistente.

El análisis de los presupuestos, las fuentes de financiación y los recursos humanos de ambas entidades subrayan esta diferencia. Mientras en la REAS, en 2010 , se contaba con un presupuesto de $221.211 €$, con un $82,4 \%$ de financiación pública y 4 personas contratadas, la XES contaba el mismo año con un presupuesto de $29.130 €$, con un $55,8 \%$ de financiación pública y una persona contratada.

La existencia de una diferencia substancial en el tipo de relación entre entidades y Administración pública entre los dos territorios analizados, que se observa claramente en la conversación con los representantes de las entidades, se traduce en la manera en que ambas entidades participan en la gobernanza y conceptualizan el cambio social que pueden promover. Entre ambos lugares, hay una disposición diferencial por parte de las entidades a la articulación formal de propuestas legislativas en la materia del fomento de la economía

3. La diferenciación entre Cataluña y la Comunidad Autónoma Vasca en las formas de relación con la Administración en materia de fomento de la economía solidaria se extiende también al análisis de las relaciones de gobernanza de las entidades orientadas al fomento de la banca ética. A pesar de ser entidades muy similares que comparten un proyecto común y tienen una relación entre ellas muy intensa, en las conversaciones con el grupo promotor del proyecto de banca ética FIARE en Vizcaya, la consideración de la posible colaboración de la administración autonómica en la constitución de una banca ética contrasta con una posición mucho más escéptica con las administraciones por parte de los responsables de COOP 57. 
alternativa y solidaria. Mientras, en el contexto vasco, la REAS Euskadi valora la interlocución con las administraciones como necesaria y normalmente fructífera, en el caso catalán, desde la XES, el cambio social se plantea a partir de una óptica más escéptica, basada en el trabajo con la sociedad civil, modificando hábitos y promoviendo el cambio desde abajo, con una confianza mucho menor en la capacidad de la gobernanza participada entre entidades y administraciones públicas para renovar las tendencias.

\section{Conclusiones}

Uno de los principales escollos con los que se encuentran los análisis de innovación social es la definición de indicadores para medir lo que es socialmente innovador o creativo. Si la literatura a este respecto ha señalado como variable determinante la modificación de las relaciones sociales entre grupos y esto apunta al tratamiento y a la transformación de las dinámicas de exclusión, el análisis de casos nos revela la complejidad que implica considerar las dimensiones económica, cultural y política del tratamiento y la transformación de las dinámicas de exclusión.

El caso de las plataformas de fomento de la economía solidaria representa un ejemplo de iniciativas que, pese a no estar directamente involucradas en la provisión de recursos y servicios a personas en situación o en riesgo de exclusión social, sí que se orientan a la articulación de cambios sociales mediante la acción sociocultural y política. Estos cambios a través de la acción indirecta inciden en las dinámicas de exclusión y el cambio en las relaciones de poder entre grupos sociales. La problemática epistemológica que se deriva de esta cuestión nos sitúa ante la caracterización de los procesos de innovación social como fenómenos sociales complejos en los que el trabajo en red -la hibridación entre actores de naturalezas distintas y en ámbitos de acción distintos- es, seguramente, el componente más eminentemente novedoso.

De modo paralelo, vemos como, en contextos locales diferentes, este tipo de plataformas tienden a comportarse de modo distinto siguiendo, entre otras variables, las trayectorias particulares y la cultura política de negociación y conflicto propios de los sistemas multinivel en los que se encuentran. Ante los retos del análisis comparativo, se subraya, pues, la importancia de considerar las herencias históricas que inciden en las relaciones de gobernanza con las que deben lidiar los actores sociales para explicar el desarrollo y la difusión de las dinámicas de innovación social. Según Van Dyck y Van der Broek (2013), la innovación social debe entenderse como un proceso territorial e institucionalmente dependiente. En los casos presentados en este artículo, el liderazgo de las entidades orientadas al fomento de la economía solidaria informa de los patrones culturales básicos del asociacionismo socialmente transformador en ambos contextos y se puede relacionar directamente con la caracterización de su participación en la gobernanza multinivel.

Junto con la cuestión de la cultura política — que incide en encauzar las relaciones entre el asociacionismo socialmente transformador y las administra- 
ciones públicas-, los procesos de transformación socioeconómica y la arquitectura institucional contra la exclusión son variables que también hay que considerar en la evaluación sobre la manera en que las dinámicas de innovación social son consideradas por parte de un sistema local de gobernanza multinivel. Aparte de la cultura política de los grupos promotores de las redes de fomento de la economía solidaria, es importante considerar el papel del sistema local de gobernanza multinivel en tanto que agente que puede colaborar en la promoción y la legitimación de las dinámicas de innovación. Las administraciones públicas pueden apelar a la participación de la sociedad civil en el tratamiento de las dinámicas de exclusión mediante la mera llamada a su colaboración, o también lo pueden hacer promoviendo políticas públicas provisoras y redistributivas que empoderen al asociacionismo socialmente comprometido. Unas políticas sociales comprometidas con la redistribución tienen un efecto evidente en la consideración de la participación en la gobernanza por parte del asociacionismo socialmente transformador como algo eficaz y efectivo.

Considerar la importancia de los sistemas locales de gobernanza en el análisis de las dinámicas de innovación social concuerda con lo que señala la literatura urbana sobre el papel de la escala local y regional en un contexto de reestructuración de las funciones gubernamentales. En un contexto de crisis multidimensional, la identificación del papel clave de los sistemas locales de gobernanza en el fomento de la innovación incorpora la atención a la manera como las sociedades locales, siguiendo patrones propios, otorgan roles determinados al mercado, a la Administración pública y a la sociedad civil en el tratamiento de las dinámicas de exclusión social y a la promoción de la transformación colectiva. En especial, la posición de la Administración pública en lo que se refiere a la regulación del mercado y a la provisión de recursos y servicios de bienestar es la de promover un estilo de relación propio con las dinámicas de innovación social.

Por lo que respecta a la influencia que pueden tener los gobiernos en tanto que facilitadores de la innovación social, la atención que ofrecen a las redes de fomento de la economía solidaria es una cuestión sensible. Al margen de la ritualización de la atención a este sector, como si de una actividad de oportunismo caritativo se tratara, los presupuestos de la economía solidaria se pueden introducir en muchas de las prácticas de la Administración pública de un modo transversal, y esta cuestión, que no pasa inadvertida por los agentes promotores de la economía solidaria, condiciona las relaciones de gobernanza a nivel político por parte del sector en cuestión. Al margen de la promoción de la innovación social como un ámbito sectorial más de las políticas públicas —más o menos marginal—, la introducción de criterios de responsabilidad social en las compras de las administraciones públicas o la difusión de los valores de la economía social y solidaria en las políticas de desarrollo local o en las políticas activas de empleo son cuestiones que determinarán la percepción de la economía solidaria como una alternativa viable en un contexto de cambio de época como el actual, y que pueden incidir positivamente en reforzar su implicación en la gestión de la complejidad. 


\section{Referencias bibliográficas}

Andreu, M. (2008). «Moviments socials i crítica al “model Barcelona” de l'esperança democràtica de 1979 al miratge olímplic de 1992 i la impostura cultural del 2004». X Coloquio Internacional de Geocritica. Barcelona: Universitat de Barcelona.

Beaumont, J. y Nichols, W. (2008). «Plural Governance, Participation and Democracy in Cities (Symposium)». International Journal of Urban and Regional Research [en línea], 32 (1), 87-94. <http://dx.doi.org/10.1111/j.1468-2427.2008.00779.x>.

Bifulco, L. y Centemeri, L. (2008). "Governance and Participation in Local Welfare: The Case of the Italian Piani di Zona». Social Policy and Administration [en línea], 42 (3), 211-227. <http://dx.doi.org/10.1111/j.1467-9515.2007.00593.x>.

Blakeley, G. (2010). "Governing Ourselves: Citizen Participation and Governance in Barcelona and Manchester». International Journal of Urban and Regional Research [en línea], 34 (1), 130-145. <http://dx.doi.org/10.1111/j.1468-2427.2010.00953.x>.

Blanco, I. y Gomà, R. (2003). "Gobiernos locales y redes participativas: Retos e innovaciones». Revista del CLAD Reforma y Democracia, 26.

BorJa, J. y Muixí, Z. (eds.) (2004). Urbanismo en el siglo XXI: Una visión crítica. Bilbao, Madrid, Valencia, Barcelona. Barcelona: Edicions UPC, ETSAB (Arquitext).

BRENNER, N. (2004). «Urban governance and the production of new state spaces in western Europe 1960-2000». Review of International Political Economy [en línea], 11 (3), 447-488. <http://dx.doi.org/10.1080/0969229042000282864>.

Capel, H. (2005). El modelo Barcelona: Un examen crítico. Barcelona: Ediciones del Serbal.

Casellas, A. (2007). «Gobernabilidad, participación ciudadana y crecimiento económico: Adaptaciones locales a estrategias globales». Scripta Nova: Revista Electrónica de Geografía y Ciencias Sociales, XI (243).

Cenicacelaya, J. (2004). "Bilbao y la urgencia de un urbanismo sostenible». En: Borja, J. y Muixí, Z. (ed.). Urbanismo en el siglo XXI: Bilbao, Madrid, Valencia, Barcelona. Barcelona: Edicions UPC.

Defourny, J. y Nyssens, M. (2013). «Social innovation, social economy and social enterprise: what can the European debate tell us?». En: Moulaert, F., MacCAllum, D., Mehmood, A., y Hamdouch, A. (ed.), The international handbook on Social Innovation. Collective action, Social Learning and Transdiciplinary Research, 40-52. Cheltenham: Edward Elgar.

Degen, M. y García, M. (eds.) (2008). La metaciudad: Barcelona. Transformación de una metrópolis. Barcelona: Anthropos.

Delgado, M. (2007). La ciudad mentirosa: Fraude y miseria del «modelo Barcelona». Madrid: Los Libros de la Catarata.

Domingo, M. y Bonet, M.R. (1998). Barcelona i els moviments socials urbans. Barcelona: Fundació Jaume Bofill / Mediterrània.

Drewe, P.; Klein, J.-L. y Hulsbergen, E. (eds.) (2008). The challenge of social innovation in urban revistalization. Amsterdam: Techne Press.

Esteban, M. (2000). Bilbao, luces y sombras de titanio. Bilbao: Servicio Editorial de la Universidad del País Vasco. 
Fung, A. (2004). Empowered participation reinventing urban democracy. Princeton, NJ: Princeton University Press.

Garcia, M. (2006). "Cítizenship Practices and Urban Governance in European Cities». Urban Studies [en línea], 43 (4), 745-765. <http://dx.doi.org/10.1080/00420980600597491>.

Gbikpi, B. y Grote, J.R. (2002). «From Democratic Government to Participatory Governance». En: Gвikpi, B. y Grote, J.R. (eds.). Participatory Governance: Political and Societal Implications. Opladen: Leske \& Budrich, 17-34.

Geddes, M. (2006). «Partnership and the Limits to Local Governance in England: Institutionalist Analysis and Neoliberalism». International Journal of Urban and Regional Research [en línea], 30 (1), 76-97. <http://dx.doi.org/10.1111/j.1468-2427.2006.00645.x>.

GonzÁlez, S. (2006). "Scalar Narratives in Bilbao: A Cultural Politics of Scales Approach to the Study of Urban Policy». International Journal of Urban and Regional Research [en línea], 30 (4), 836-857. <http://dx.doi.org/10.1111/j.1468-2427.2006.00693.x>.

González, S. y Healey, P. (2005). «A Sociological institutionalist approach to the study of innovation in governance capacity». Urban Studies [en línea], 42 (11), 2055-2069. <http://dx.doi.org/10.1080/00420980500279778>.

Jessop, B. (2002). "Governing cities and regions: Territorial restructuring in a global age». En: Scotт, A.J. (ed.). Global city-regions: Trends, theory and policy. Oxford: Blackwell.

- (2004). «Governance, multi-level governance and metagovernance». En: BACHE, I. (ed.). Multi-level Governance. Oxford: Blackwell.

LE GALÈs, P. (2002). European cities, social conflicts and governance. Oxford: Blackwell.

LÉVEsque, B. (2013). «Social innovation in governance and public management systems: Towards a new paradigm?». En: MoulaERT et al. (eds.). Social innovation: Collective action, Social learning and Transdisciplinary research. Cheltenham: Edward Publishing, 25-39.

MacCallum, D.; Moulaert, F.; Hillier, J. y Vicari, S. (2009). Social Innovation and Territorial Development. Oxon: Ashgate Publishing.

MacLeod, G. (2001). «New Regionalism Reconsidered: Globalization and the Remaking of Political Economic Space». International Journal of Urban and Regional Research [en línea], 25 (4), 804-829. <http://dx.doi.org/10.1111/1468-2427.00345>.

Maloutas, T. y Pantelidou, M. (2004). «The Glass Menagerie of Urban Governance and Social Cohesion: Concepts and Stakes / Concepts as Stakes». International Journal of Urban and Regional Research [en línea], 28 (2), 449-465. <http://dx.doi.org/10.1111/j.0309-1317.2004.00528.x>.

Mingione, E. (1991). Fragmented Societies. Oxford: Blackwell.

Montaner, J.M. (2004). «La evolución del modelo Barcelona (1979-2002)». En: BorJa, J. y MuIxí, Z. (ed.). Urbanismo en el siglo XXI: Una visión crítica. Bilbao, Madrid, Valencia, Barcelona. Barcelona: Edicions UPC/ETSAB.

Mouffe, C. (2000). The democratic paradox. Londres: Verso.

Moulaert, F.; MacCallum, D.; Mehmood, A. y Hamdouch, A. (2013). Social innovation: Collective action, Social learning and Transdisciplinary research. Cheltenham: Edward Elgar Publishing.

PARÉs, M. (2009). Participación ciudadana y calidad democrática: Evaluando las nuevas formas de democracia participativa. Barcelona: Ariel. 
Plaza, B. (2006). "The Return on Investment of the Guggenheim Museum Bilbao». International Journal of Urban and Regional Research [en línea], 30 (2), 452-467. <http://dx.doi.org/10.1111/j.1468-2427.2006.00672.x>.

- (2008). «On Some Challenges and Conditions for the Guggenheim Museum Bilbao to be an Effective Economic Re-activator». International Journal of Urban and Regional Research [en línea], 32 (2), 506-517. <http://dx.doi.org/10.1111/j.1468-2427.2008.00796.x>.

Rhodes, R. (1997). Understanding Governance: Policy Networks, Governance, Reflexivity and Accountability. Buckingham: Open University Press.

Rodríguez, A. (2002). «Reinventar la ciudad: Milagros y espejismos de la revitalización urbana en Bilbao». Lan Harremanak, 6, 69-108.

Silver, H.; Scott, A. y Kazepov, Y. (2010). «Participation in Urban Contention and Deliberation». International Journal of Urban and Regional Research [en línea], 34 (3), 453-477. <http://dx.doi.org/10.1111/j.1468-2427.2010.00963.x>.

Sommers, M. (2005). «Let them Eat Social Capital: Socializing the Market versus Marketizing the Social». Thesis Eleven [en línea], 81 (mayo), 5-19. <http://dx.doi.org/10.1177/0725513605051611>.

Swyngedouw, E. (2005). "Governance, innovation and the citizen: The Janus face of governance-beyond-the-state». Urban Studies [en línea], 42 (11), 1991-2006. <http://dx.doi.org/10.1080/00420980500279869>.

Uitermark, J. y Duyvendak, J.W. (2008). "Citizen Participation in a Mediated Age: Neighbourhood Governance in The Netherlands». International Journal of Urban and Regional Research [en línea], 32 (1), 114-134. <http://dx.doi.org/10.1111/j.1468-2427.2007.00743.x>.

Unió Temporal d’Escribes (UTE) (2004). Barcelona, marca registrada: Un model per desarmar. Barcelona: Virus.

URrutia, V. (1992). «Transformación y persistencia de los movimientos sociales urbanos». Política y Sociedad [en línea], 10, 49-66.

- (2004). «Bilbao, el peso de un contexto». En: Borja, J. y Muixí, Z. (ed.). Urbanismo en el siglo XXI. Barcelona: Universitat Politècnica de Catalunya, 51-62.

Van Dyck, B., y Van den Broeck, P. (2013). «Social innovation: a territorial process». En: Moulaert, F., MacCallum, D., Mehmood, A., y Hamdouch, A. (ed.). The international handbook on Social Innovation. Collective action, Social Learning and Transdiciplinary Research, 131-141. Cheltenham: Edward Elgar. 